\title{
Optical Imaging Technology for Real-time Tumor Monitoring
}

Yoo-kyoung Shin

Joo Beom Eom

Department of Biomedical Science, College of Medicine, Dankook University, Cheonan, Korea

Received July 20, 2021

Accepted August 11, 2021

\footnotetext{
Correspondence

Joo Beom Eom

Department of Biomedical Science, College of Medicine, Dankook University, 119 Dandae-ro, Dongnam-gu, Cheonan 31116, Korea

Tel.: +82-41-550-3275

Fax: +82-41-550-3275

E-mail: jbeomवdankook.ac.kr

(C) Korean Society for Laser Medicine and Surgery

(c) This is an open access article distributed under the terms of the Creative Commons Attribution NonCommercial License (http://creativecommons.org/ licenses/by-nc/4.0) which permits unrestricted noncommercial use, distribution, and reproduction in any medium, provided the original work is properly cited.
}

Optical imaging modalities with properties of real-time, non-invasive, in vivo, and high resolution for image-guided surgery have been widely studied. In this review, we introduce two optical imaging systems, that could be the core of image-guided surgery and introduce the system configuration, implementation, and operation methods. First, we introduce the optical coherence tomography (OCT) system implemented by our research group. This system is implemented based on a sweptsource, and the system has an axial resolution of $11 \mu \mathrm{m}$ and a lateral resolution of $22 \mu \mathrm{m}$. Second, we introduce a fluorescence imaging system. The fluorescence imaging system was implemented based on the absorption and fluorescence wavelength of indocyanine green (ICG), with a light-emitting diode (LED) light source. To confirm the performance of the two imaging systems, human malignant melanoma cells were injected into BALB/c nude mice to create a xenograft model and using this, OCT images of cancer and pathological slide images were compared. In addition, in a mouse model, an intravenous injection of indocyanine green was used with a fluorescence imaging system to detect real-time images moving along blood vessels and to detect sentinel lymph nodes, which could be very important for cancer staging. Finally, polarization-sensitive OCT to find the boundaries of cancer in real-time and real-time image-guided surgery using a developed contrast agent and fluorescence imaging system were introduced.

\section{Key words}

Optical imaging; Optical coherence tomography; Near-infrared fluorescence; Indocyanine green; Cancer 


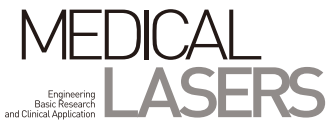

\section{INTRODUCTION}

Despite many years of basic and clinical research, cancer is a major health problem worldwide. ' Early diagnosis of growing cancer and rapid treatment is key to extend the life and improve the quality of life. Different imaging modalities such as computed tomography (CT), magnetic resonance imaging (MRI), positron emission tomography (PET), single-photon emission computed tomography (SPECT), and ultrasound imaging (UI) have been used to aid clinicians in the diagnosis, staging, and treatment of human cancers. ${ }^{2}$ However, although this method is excellent for diagnosing cancer, it is difficult to detect early cancer due to its low resolution, and it is difficult to accurately remove the lesion during surgery, resulting in many side effects.

Therefore, many research groups have showed a lot of interest in research for early detection of cancer and realtime identification of lesions during surgery, and studied optical imaging as an alternative to solve them. Compared established cancer imaging modalities, optical imaging has high resolution to probe functional and structural changes in real time and is a in vivo and noninvasive method, which can protect patient from potential hazard of non-optical methods le.g., biopsy and subsequent histologic examination) and harmful radiation., ${ }^{2,3}$ These optical imaging methods include confocal imaging, optical molecular imaging, optical coherence tomography (OCT), and near-infrared (NIR) fluorescence imaging among several others. ${ }^{4}$

Among them, OCT and fluorescence imaging are representative methods that can identify the boundaries of cancer in real time and identify lesions during surgery. OCT is well known for retinal imaging in ophthalmology $y^{5}$ and studies have shown that OCT has improved diagnostic efficacy in various structures such as the skin, gastrointestinal, respiratory, genitourinary tracts and the oral cavity. ${ }^{5-13}$ Recently, OCT has been proposed for the early detection of cancer by distinguishing tumorous and nontumorous tissues with its high resolution at large lup to 2 to $3 \mathrm{~mm}$ ) penetration depths. OCT can be used guided biopsy and can aid in the intraoperative imaging of cancer by providing real-time feedback to surgeons. It can also be used for monitoring of tumor responses to treatments le.g., radiotherapy, chemotherapy, photodynamic therapy (PDT), and ablative (thermal- or cryo-) therapy. ${ }^{2,14-16}$

Fluorescence imaging (FI) is one of the most popular method for the visualization of cells, tissues, and biological processes taking place in a living organism. Fluorescence images can be produced from microscopy, imaging probes, and spectroscopy. ${ }^{17,18}$ In particular, near-infrared (NIR) fluorescence imaging has been playing a significant role in the field of tumor-specific fluorescence-guided surgery (TS-FGS). ${ }^{19}$ NIR fluorescence provides high-resolution images and penetrates into blood and tissues more than a few hundred microns since the optical window of 650 to $1350 \mathrm{~nm}$ has maximum depth of penetration in tissues and relatively low scattering and autofluorescence of biomolecules. With these advantages, NIR fluorescence imaging can provide real-time image guidance to surgeons for finding structures that need to be resected, such as tumor margins and sentinel lymph nodes. ${ }^{20}$

The purpose of this review is to introduce the operating principle of the OCT and fluorescence imaging systems mentioned above in the optical imaging system, which is the core for image guided surgery, and the applicability of the system by making an animal model. First, an introduction of the implemented OCT and an animal model injected with human malignant melanoma cell were evaluated, and the effectiveness of the OCT was evaluated and compared with pathological data. Second, the construction and operation of the fabricated fluorescence imaging system were introduced. Using the implemented system, indocyanine green (ICG) was injected intravenously into a mouse model and the performance of the system was confirmed through real-time images moving along the blood vessels. In addition, using a fluorescence imaging system, the possibility of use in surgery was confirmed in real time through the detection of sentinel lymph nodes, which can be very important for cancer staging. Finally, we would like to confirm the importance of the research group's image guided surgery by introducing the latest research trends in optical imaging.

\section{METHOD}

\section{Optical coherence tomography system}

Fig. 1 depicts the swept-source optical coherence tomography (SS-OCT) system, which was constructed based on a Mach-Zehnder interferometer. The system consists of five modules: a wavelength swept source, an optical fiber interferometer, a scanner, a controller, and a digitizer, used for acquiring and displaying OCT volume images. In order to use the OCT system to identify lesions during surgery, a fast scanning speed is required. Therefore, in this system, a wavelength-swept laser source (SL132120; Thorlabs Inc., Newton, NJ, USA) with a center wavelength of $1310 \mathrm{~nm}$, a maximum half width of $100 \mathrm{~nm}$, and a scan rate of $200 \mathrm{kHz}$ was used as the light source. The laser beam from the swept source was divided into 


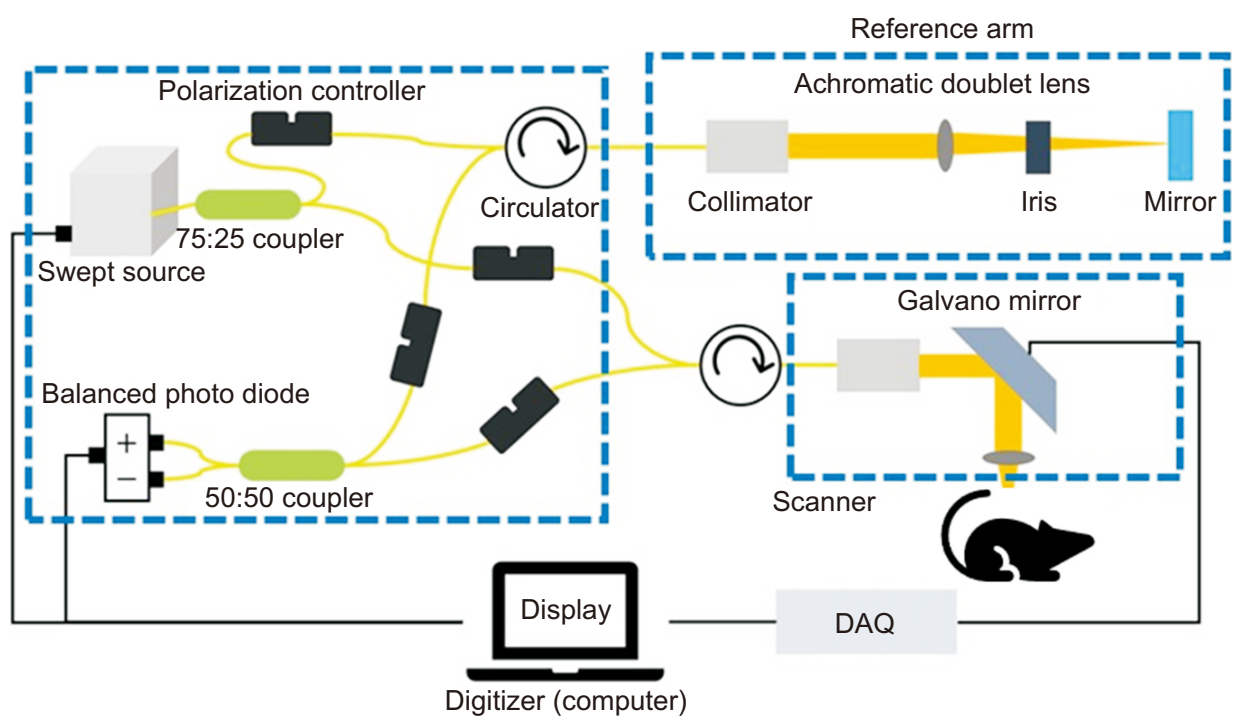

Fig. 1. Schematic of swept-source optical coherence tomography (SS-OCT) system with scan probe. DAQ, data acquisition. a sample arm and reference arm through a 75:25 fiber coupler (TW1300R3F2; Thorlabs), which was guided to the optical fiber Mach-Zehnder interferometer. Fig. 1 shows a sample arm irradiated light to the sample, and the sample arm was fabricated with a Galvo-mirror and scan lens (LSM54-1310; Thorlabs) using a 3-D printer. The reference arm consists of a collimator, lens, iris, and mirror, and this was used as the reference beam. And the iris was used to control to have the appropriate optical power in the reference arm. After the light is reflected from the sample and the reference arm, the lights interfered with each other through a 50:50 coupler. This interference signal was detected by the balanced photodetector. The spectral interference signal which was converted into a voltage was transmitted to a 12-bit high-speed digitizer. To implement the fast Fourier transform, the spectrum interpolation was previously performed on all A-scans for resampling $\mathrm{k}$-space. The number of A-scans samples was 500 and the scan range of the system was $12 \mathrm{~mm} \times$ $12 \mathrm{~mm}$. The 2-D cross-sectional image was constructed by using 512 B-scans and the $3-D$ volume images utilized 512 cross-sectional images. The axial resolution and lateral resolution are $11 \mu \mathrm{m}$ and $22 \mu \mathrm{m}$ in the air.

\section{Fluorescence imaging camera based on light- emitting diode (LED) lightening}

A schematic diagram and image of the fluorescence imaging system with LED lightening is shown in Fig. 2. The fluorescence imaging system consisted of LED lightening, camera module with lens and band pass filter. As the wavelength of the LED, an LED having a wavelength of $780 \mathrm{~nm}$, which is the absorption wavelength of ICG, was used. In addition, as the band pass filter, a long pass filter

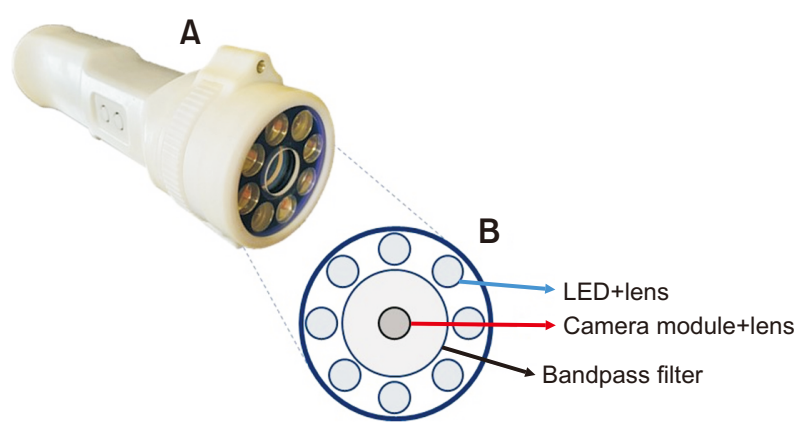

Fig. 2. Fluorescence imaging camera based on light-emitting diode (LED) lightening ((A) A picture of implemented fluorescence imaging camera, (B) schematic of lightening and camera module part of fluorescence imaging system with LED).

that passes over $800 \mathrm{~nm}$, the fluorescence wavelength of $I C G$, was used. To secure the entire image of the mouse model, a lens with a focal length of about $200 \mathrm{~mm}$ was used and mounted on the front of the camera module. In order to deliver a constant light to the sample, a reflector was manufactured and a lens was attached to each LED light to prevent light from spreading. Images acquired from the camera using a USB 3.0 port were signal acquired using a graphical user interface (GUI).

\section{Animal model}

A total of 5 male Balb/c nude mice laged 7 weeks) used in the experiment were approved by the Animal Protection Committee of Dankook University. To confirm realtime fluorescence and OCT images of lesions, mice were inoculated at two spots on the dorsal flank with 1×106 human malignant melanoma A-375 cells, diluted in $100 \mu \mathrm{L}$ phosphate-buffered saline. The A-375 cells were grown 
and maintained in minimum essential medium (MEM) supplemented with $10 \%$ fetal bovine serum and $1 \%$ penicillin and streptomycin. Subcutaneous tumor growth and overall health were monitored twice a week. In vivo imaging experiments were performed 3-4 weeks after A-375 cells injection. For OCT imaging and ICG NIR imaging, animals were anesthetized with Zoletil $50130 \mathrm{mg} / \mathrm{kg}$; Tiletamine and zolazepam) and Rompun (10 mg/kg; Xylazine).

\section{Description and specification of ICG}

Indocyanine green is a cyanine dye having a molecular weight of $774.97 \mathrm{~g} / \mathrm{mol}$. It is a negatively charged ion that belongs to the cyanine dyes (Fig. 3B). ${ }^{21}$ ICG (BioActs, RFP0815) was dissolved in dimethyl sulfoxide (DMSO) and diluted with PBS to the final concentration $10 \mu \mathrm{M}$ for the experiments. To accurately measure the fluorescence wavelength for the absorption spectrum of ICG presented in the specification, $780 \mathrm{~nm}$ laser diode (LD) as shown in Fig. $3 \mathrm{C}$ was used, and the fluorescence spectrum was measured using the implemented fluorescence spectroscopy system. The wavelength range at full width half maximum of the measured fluorescence spectrum was $810 \mathrm{~nm}$ to $860 \mathrm{~nm}$ (Fig. 3D), confirming that it was a fluorescence signal suitable for the characteristics of the 800 $\mathrm{nm}$ long pass filter in the fabricated system.

\section{RESULTS}

\section{Optical coherence tomography structural imag- ing of mouse dorsal skin}

Subcutaneous tumor was imaged with OCT system 12 days after injection. 3D volume and $2 \mathrm{D}$ cross-sectional images of cancerous region (Fig. 4D, F, G) were compared with the images of normal region (Fig. 4A-C). The cancerous tissue appears darker in the OCT image in part because it contains more blood than normal tissue. The OCT system used in this study employs a $1310 \mathrm{~nm}$ light source. At this wavelength, light absorption by blood is considerable, which produces the darker appearance of the malignant tissue. ${ }^{22}$ Additional differences between normal and cancerous regions was shown through statistical analysis of OCT images and corresponding histological sections (Fig. 5).

\section{Analysis of corresponding H\&E-stained tissue sections and OCT images}

The red and yellow rectangles marked on the Fig. 5A correspond to histological images (Fig. 5B, C) and compared with each other. Histological images were taken using cross-sectional slicing and hematoxylin \& eosin (H\&E) staining to confirm the correlation with the OCT image taken previously. Generally normal tissue has wellorganized tissue structure, while the structure of cancer tissue is often disorganized. ${ }^{2}$ Fig. 5 shows the different

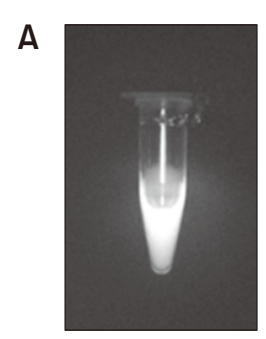

C

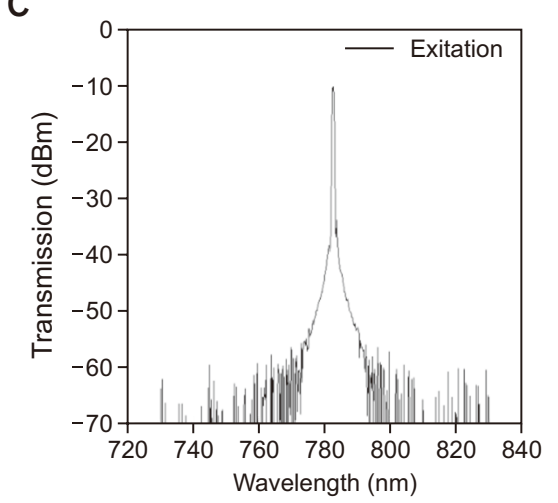

B<smiles></smiles>

D

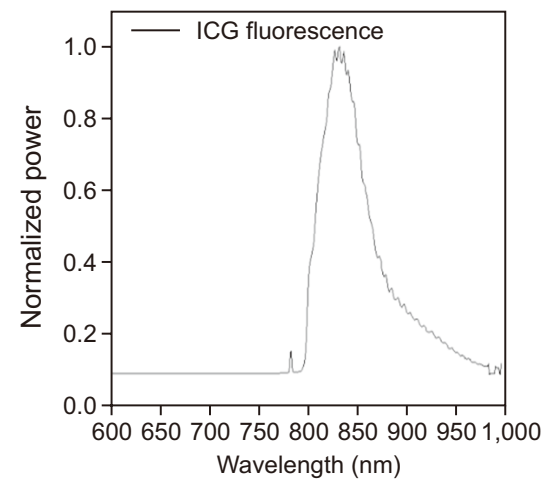

Fig. 3. Description and specification of indocyanine green (ICG) ((A) 10 $\mu \mathrm{M}$ ICG imaged by implemented near infrared (NIR) fluorescence imaging camera, (B) Chemical structure of ICG, (C) The wavelength of excitation light, (D) ICG fluorescence wavelength). 

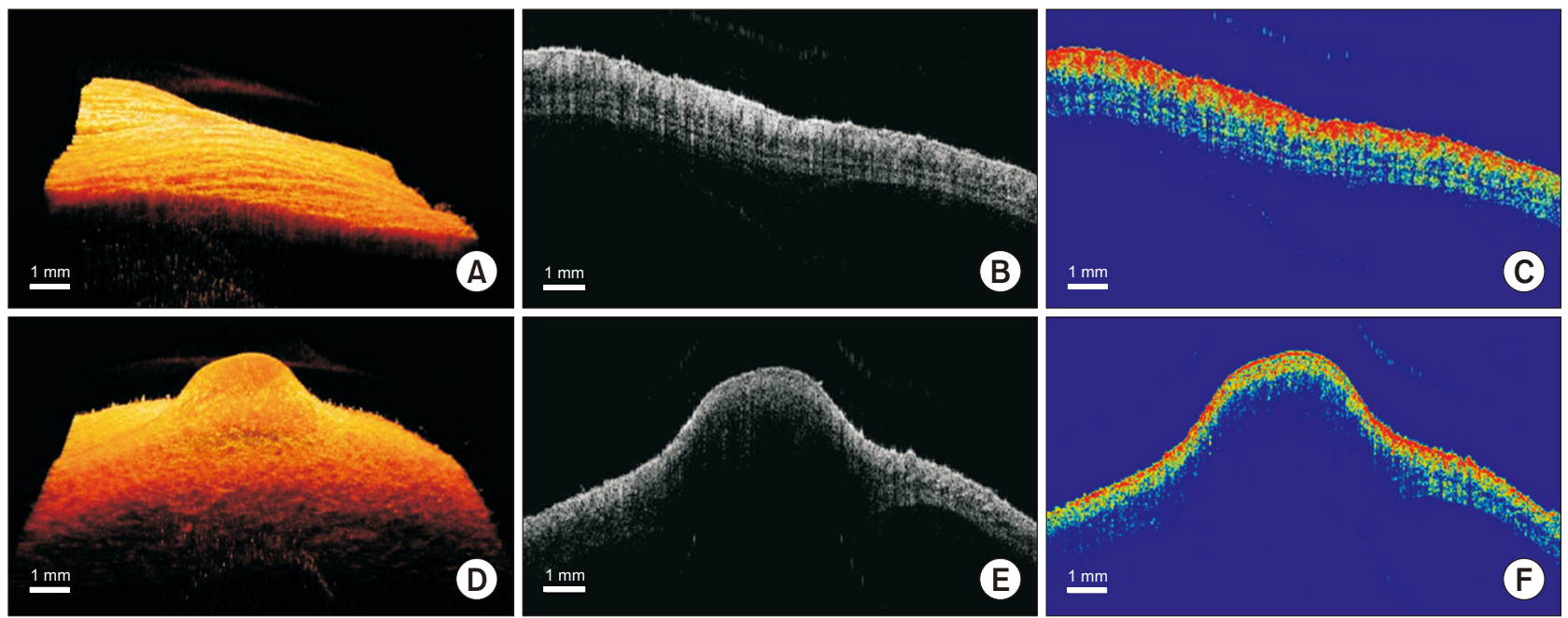

Fig. 4. OCT images of normal and cancerous region in BALB/c nude mouse ((A-C) normal region, (D-F) cancerous region, (A, D) 3D volume images, (B, E) 2D cross-sectional images, and (C, F) corlor-coded images). Scale bars represent $1 \mathrm{~mm}$.

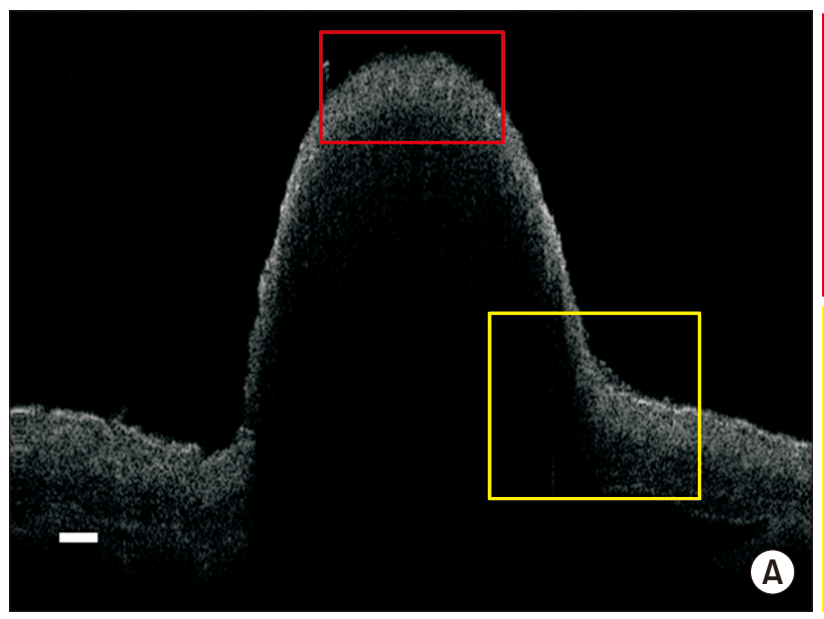

characteristic organization and thickness of skin layers. Hypodermis, containing adipose tissue and muscle was below the dermis in normal region (Fig. 5C). In contrast, hypodermis except the muscle layer has become too thin to be seen in cancerous region (Fig.5B). These results suggest that OCT can provide diagnostic information for precancerous lesions by detecting morphological changes. $^{22}$

However, the intensity image of OCT has a limitation in being able to identify the clear boundaries of cancer when compared with images obtained using pathology slides. Therefore, recently, many research groups are actively conducting research on diagnosing cancer boundaries in real time using polarization-sensitive OCT using polarization technique. ${ }^{23}$ Polarization-sensitive (PS) OCT is an imaging modality that provides large volumetric

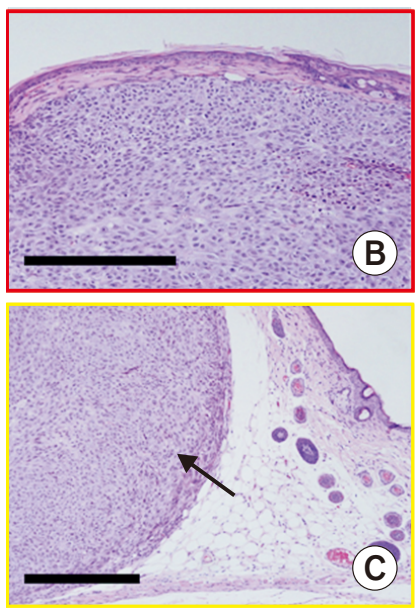

Fig. 5. In vivo OCT image of tumor and corresponding hematoxylin \& eosin-stained tissue section. The red rectangle indicates only the area where cancer occurs, and the yellow rectangle indicates the boundary between tumor and normal tissue. ((A) 2D OCT image of tumor, (B, C) corresponding histology of OCT image in (A). The black arrow points out the tumor mass. Scale bar represents $500 \mu \mathrm{m}$ in (A) and 200 $\mu \mathrm{m}$ in Fig. (B, C). views of tissue microstructure with high resolution while simultaneously measuring birefringence of organized tissues such as collagen. ${ }^{24}$ PS-OCT clearly shows a distinction between solid carcinoma and surrounding fibrosis compared to structural OCT (Fig. 6A, B). The boundary between solid carcinoma and fibrosis clearly visualized with PS-OCT is confirmed with corresponding pathological slide images (Fig. 6C). These results suggest that PSOCT could serve as a powerful imaging technology for assessing tissue acquisition sites within lung nodules by drastically enhancing differentiation between tumor and fibrosis. This work demonstrates the significant potential of using PS-OCT for biopsy guidance and enhanced cancer detection in the operation room. ${ }^{25}$ 


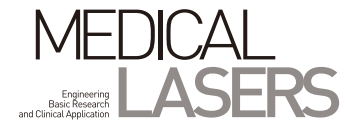

\section{In vivo imaging of ICG under mouse model}

Fig. 7 depicts a fluorescence signal distribution in the mouse body after intravenous injection of ICG (150 $\mu$ of 10 $\mu M$ ICG). From 5 minutes to 6 hours, the signals seems to gradually accumulate towards the liver, intestine, and after 6 hours, started to accumulate to the kidneys (Fig. 7A). 6 hours after the injection of ICG, we extracted or-

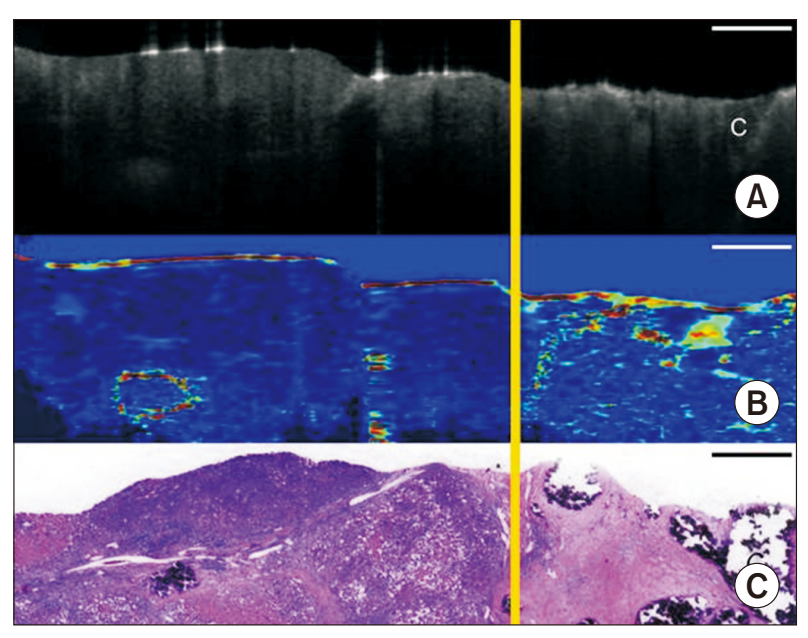

Fig. 6. Polarization-sensitive (PS) OCT of poorly differentiated carcinoma with surrounding fibrosis in ex vivo lung. (A) Structural OCT does not clearly show a distinction between solid carcinoma and adjacent fibrosis. Calcifications within the fibrosis can be seen as signal-poor structures (C). (B) PS-OCT shows a clear delineation between solid carcinoma (left of line) and fibrosis (right of line), with no birefringence signal in the regions of carcinoma and high birefringence signal in the regions of fibrosis. (C) Matched histology confirming the demarcation between carcinoma and fibrosis. Scale bars: $1 \mathrm{~mm}$. Figure reprinted with permission from Reference 39. gans from a mouse model and checked the intensity of ICG respectively. The level of ICG emitted from liver was the most highly detected, followed by small intestine and kidneys (Fig. 7B). Xenograft tumors in mice could hardly be detected by free ICG because of its rapid clearance and low accumulation in the tumor. ${ }^{26}$

To overcome these limitations, incorporating ICG into nanoparticle (NP) platforms is extensively studied. Various types of ICG-incorporated NPs have been developed and functionalized to embrace imaging and therapeutic techniques for cancer diagnosis and treatment. These ICG NPs show enhanced photostability, biocompatibility and tumor accumulation, low self-aggregation, and usually brighter fluorescence signal compared to free ICG dye due to the protective architecture. ${ }^{27}$ Zheng et al. ${ }^{28}$ prepared biodegradable folic acid-targeted NPs encapsulating ICG (FA-INPs) with intrinsic FA-targeting ligands. The FA-INP showed enhanced ICG stability, produced stronger temperature response than free ICG and exhibited significant targeting to breast tumors in in vivo studies as shown in Fig. 8. This makes the system promising as a theranostic agent for imaging guided cancer photothermal therapy clinically.

\section{Near-infrared lymphatic imaging using ICG in mice}

One example of intraoperative fluorescence imaging is sentinel lymph node (SLN) biopsy (SLNB) using fluorescent probes. So we generate xenograft models of malignant melanoma cancer by dorsal flank injection of A-375 cells to confirm the possibility of detection of

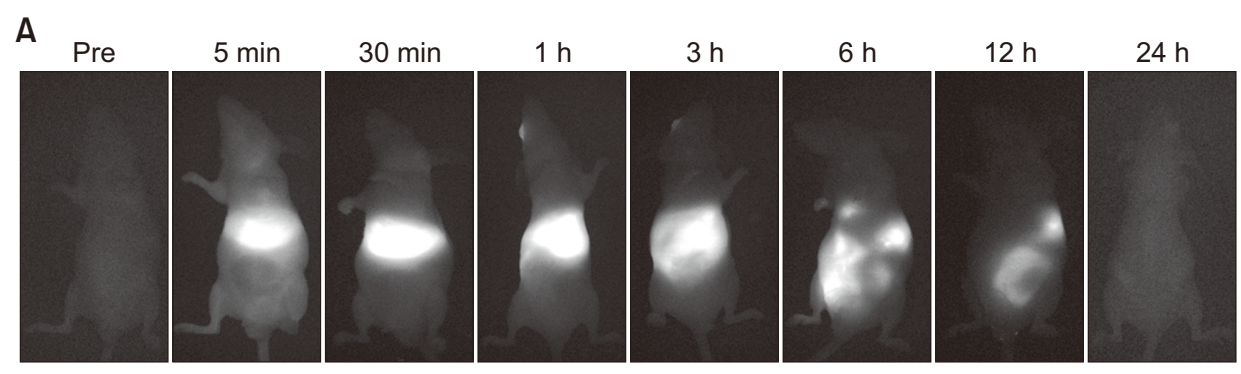

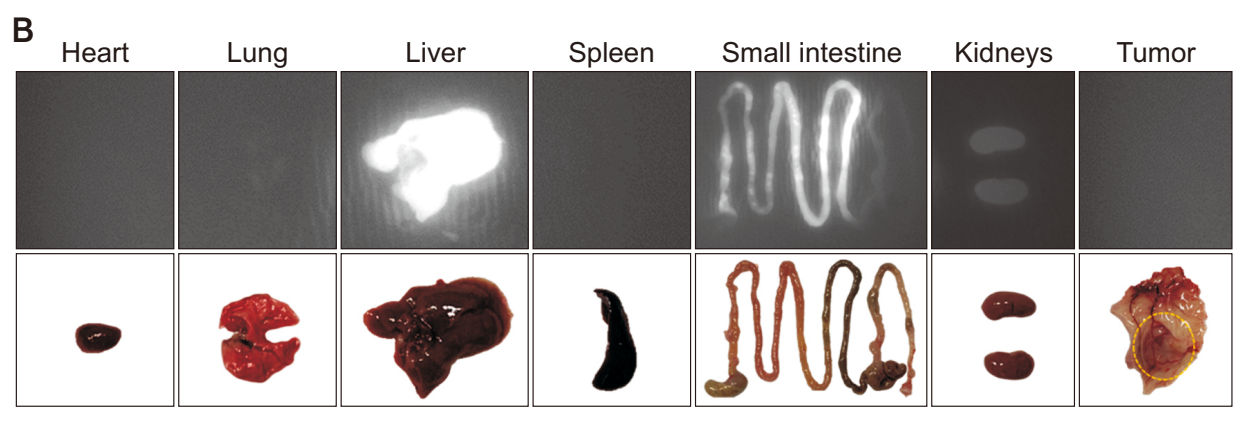

Fig. 7. In vivo NIR fluorescence (NIRF) imaging of ICG in nude mice bearing A-375 tumors after intravenous injection of ICG. (A) Time-lapse NIRF images of a mouse model, (B) NIRF images of organs 6 hour post-injection of ICG. 
A

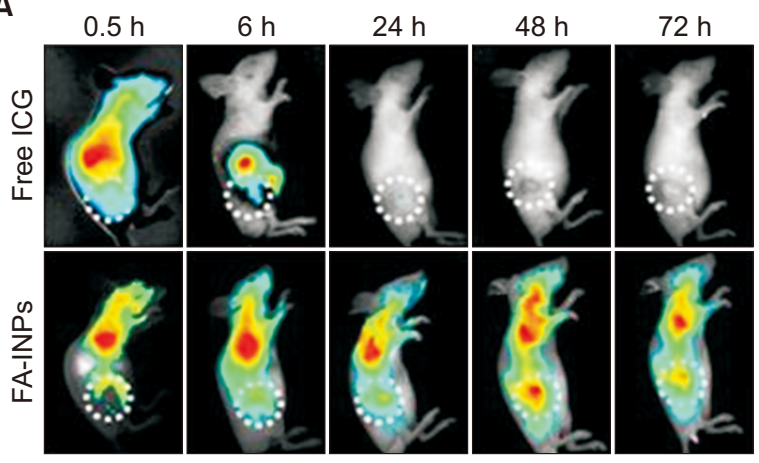

B

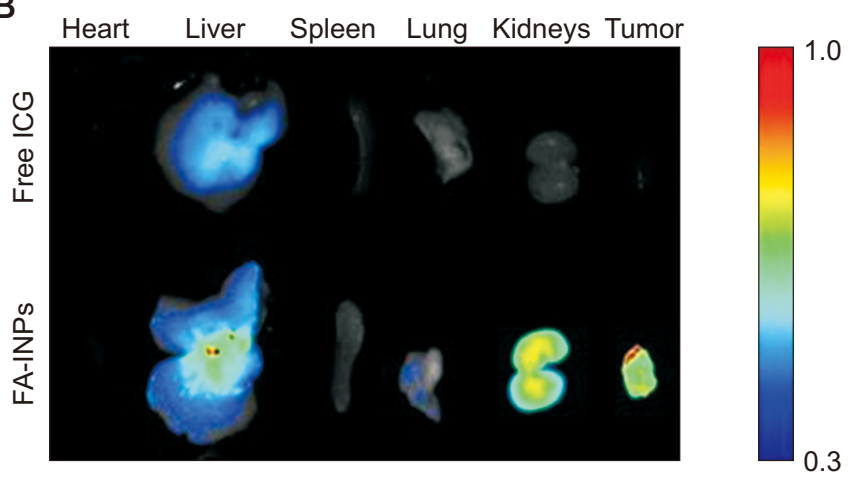

Fig. 8. In vivo NIRF imaging of FA-INPs and free ICG in nude mice bearing MCF-7 tumors after intravenous injection. (A) Time-lapse NIRF images of nude mice (the tumors are circled with a dotted line) (B) NIRF images of organs and tumors $48 \mathrm{~h}$ post-injection of free ICG and FA-INPs. Figure reprinted with permission from Reference 24.
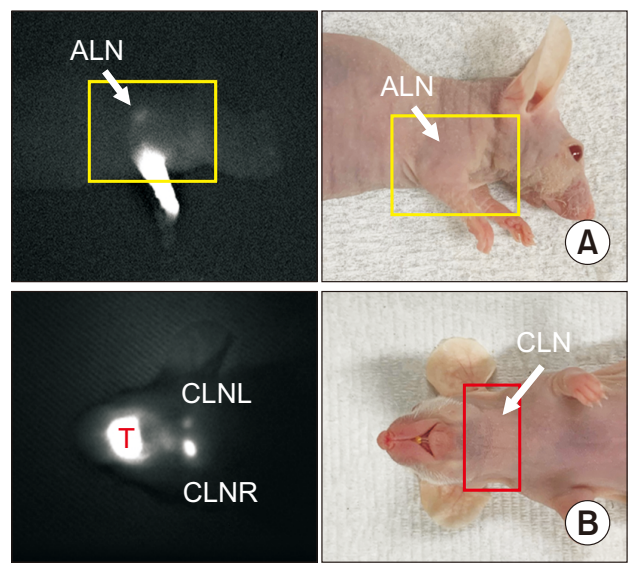

(B)

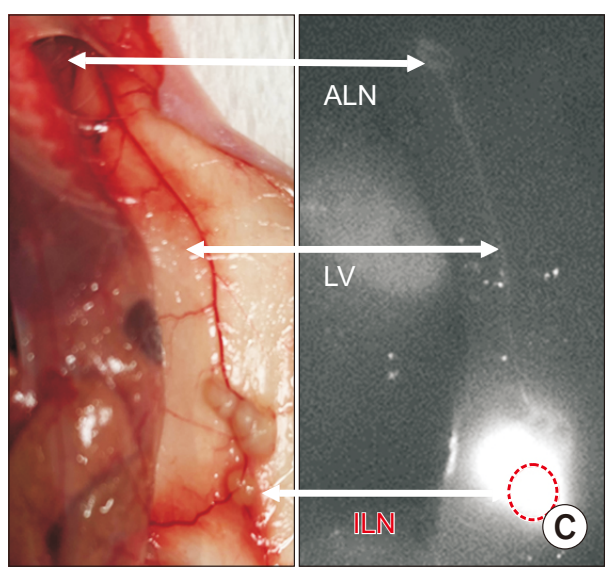

Fig. 9. Sentinel lymph node detection using NIR fluorescent ICG in mice. (A) Axillary sentinel lymph node identified after injection of ICG in the fore paw, (B) Cervical sentinel lymph node identified after injection of ICG in the tongue, $(\mathrm{C})$ Lymphatic route from the ILN to the ALN. ALN, axillary sentinel lymph node; T, tongue; CLNL, cervical sentinel lymph node left; CLNR, cervical sentinel lymph node right; LV, lymphatic vessel; ILN, inguinal lymph node. sentinel lymph nodes in real time. 3 weeks after tumor cell injection, in vivo imaging were performed after injection of ICG into a footpad, a tongue, and ILN of xenograft model. Fig. 9A, B show the diagnostic capability of ICG to detect lymph nodes. Lymph nodes are the initial site for metastases for most cancers. The sentinel lymph node is the lymph node that receives the first lymph flow from a primary malignant tumor. The detection of the sentinel lymph node is vital for staging cancer and affects the choice of proper therapy related to the survival rates. The primary tumor and metastatic lymph node should be removed during the surgical operation. Currently attractive method ICG is used to detect lymph nodes and to harvest sentinel lymph nodes. ${ }^{17,29}$ In breast cancer, dissection of axillary lymph node that filter fluid draining from the primary tumor is important for cancer staging. ${ }^{3,30}$ Adsorption of ICG to human serum albumin is effective for SLN mapping in breast cancer. ${ }^{31}$ We injected ICG into the upstream lymph node ILN and checked the flow of ICG moved to downstream lymph node ALN (Fig. 9C). This result shows drugs injected into upstream lymph nodes can reach metastatic lymph nodes for prevention and treatment of metastatic lymph nodes.

\section{CONCLUSIONS AND DISCUSSIONS}

We presented an overview of the optical imaging method capable of real-time monitoring of lesions and their applications. In order to acquire a real-time image of the lesion, a high-speed OCT having a speed of $400 \mathrm{fps}$ was implemented using a high-speed swept source. In consideration of high-speed scanning of samples and convenience, a scanner was implemented using Galvomirror and 3D printer. In addition, a fluorescence imaging system optimized for the absorption or fluorescence wavelength of ICG, a representative contrast agent, was prepared for real-time fluorescence imaging. Considering user convenience, it is implemented as a hand-held type, and $780 \mathrm{~nm}$ LED is used as the light source.

To confirm the performance and surgical application 
of both systems, we prepared xenograft mouse models injected with human malignant melanoma cell and successfully acquired images using both imaging systems. Through real-time OCT images of lesions acquired in the xenograft mouse model, we saw the possibility of distinguishing between normal and cancerous tissues. However, it is difficult to determine the exact boundary of cancer based only on the intensity images of OCT, so many other research groups are conducting research on the boundary of cancer in real time during surgery using the OCT system using polarization. Therefore, we think that the real-time image confirmation of the lesion for OCT performed by this research group and the OCT technology using the polarization technique conducted by other research groups suggested the possibility of real-time classification of cancer boundaries in three dimensions. It is expected to be used in clinical practice in the future.

In addition, using ICG, a fluorescent agent widely used in clinical practice, ICG images were checked hourly through intravenous injection, and images of sentinel lymph nodes were acquired. ICG, a fluorescent agent, moved along the blood vessels, and it could be seen from the image that it was accumulated or escaped from each organ over time. This proved to be a key technology for image guided surgery as it enables real-time imaging of fluorescent agents during surgery. However, since ICG is not a tumor-specific agent, tumors generated in the xenograft mouse model could hardly be detected. However, as many research groups have recently introduced in this review, there are many studies on the development of tumor-specific agents and their application to image guided surgery.

As suggested, optical imaging systems, especially OCT systems and fluorescence imaging systems, can check non-invasive and in vivo images in real time, so it is clear that they have great potential in image guided surgery as presented in this review. Recently, as interest in minimally invasive surgery that emphasizes quality of life and minimizes side effects increases, image-guided surgery is emerging in the medical device field. Therefore, the two optical imaging techniques presented in this review are expected to grow as core technologies in related fields.

\section{CONFLICT OF INTEREST}

No potential conflict of interest relevant to this article was reported.

\section{FUNDING}

This work was supported by the Korea Medical Device Development Fund (KMDF) grant funded by the Korea government (KMDF_PR_20200901_0024). All experiments with mice were performed in the animal facility at Dankook University according to the guidelines of the Dankook University Institutional Animal Care \& Use Committee.

\section{REFERENCES}

1. Sleeman J, Steeg PS. Cancer metastasis as a therapeutic target. Eur J Cancer 2010;46:1177-80.

2. Wang J, Xu Y, Boppart SA. Review of optical coherence tomography in oncology. J Biomed Opt 2017;22:1-23.

3. Rajabi-Estarabadi A, Bittar JM, Zheng C, Nascimento V, Camacho I, Feun LG, et al. Optical coherence tomography imaging of melanoma skin cancer. Lasers Med Sci 2019;34:411-20.

4. Balas C. Review of biomedical optical imaging-a powerful, non-invasive, non-ionizing technology for improving in vivo diagnosis. Meas Sci Technol 2009;20:104020.

5. Wojtkowski M, Bajraszewski T, Gorczyńska I, Targowski P, Kowalczyk A, Wasilewski W, et al. Ophthalmic imaging by spectral optical coherence tomography. Am J Ophthalmol 2004;138:412-9.

6. Leitgeb RA. En face optical coherence tomography: a technology review [Invited]. Biomed Opt Express 2019;10:2177-201.

7. Schmitt JM, Yadlowsky MJ, Bonner RF. Subsurface imaging of living skin with optical coherence microscopy. Dermatology 1995;191:93-8.

8. Kobayashi K, Izatt JA, Kulkarni MD, Willis J, Sivak MV Jr. Highresolution cross-sectional imaging of the gastrointestinal tract using optical coherence tomography: preliminary results. Gastrointest Endosc 1998;47:515-23.

9. Colston BW Jr, Everett MJ, Da Silva LB, Otis LL, Stroeve P, Nathel $\mathrm{H}$. Imaging of hard- and soft-tissue structure in the oral cavity by optical coherence tomography. Appl Opt 1998;37:35825.

10. Tearney GJ, Brezinski ME, Southern JF, Bouma BE, Boppart SA, Fujimoto JG. Optical biopsy in human urologic tissue using optical coherence tomography. J Urol 1997;157:1915-9.

11. Zysk AM, Nguyen FT, Oldenburg AL, Marks DL, Boppart SA. Optical coherence tomography: a review of clinical development from bench to bedside. J Biomed Opt 2007;12:051403.

12. Jesser CA, Boppart SA, Pitris C, Stamper DL, Nielsen GP, Brezinski ME, et al. High resolution imaging of transitional cell carcinoma with optical coherence tomography: feasibility for the evaluation of bladder pathology. Br J Radiol 1999;72:11706.

13. Gambichler T, Orlikov A, Vasa R, Moussa G, Hoffmann K, 
Stücker $M$, et al. In vivo optical coherence tomography of basal cell carcinoma. J Dermatol Sci 2007;45:167-73.

14. Gladkova N, Maslennikova A, Balalaeva I, Feldchtein F, Kiseleva E, Karabut M, et al. Application of optical coherence tomography in the diagnosis of mucositis in patients with head and neck cancer during a course of radio(chemo)therapy. Med Lasers Appl 2008;23:186-95.

15. García-Layana A, Salinas-Alamán A, Maldonado MJ, Sainz-Gómez C, Fernández-Hortelano A. Optical coherence tomography to monitor photodynamic therapy in pathological myopia. $\mathrm{Br} \mathrm{J}$ Ophthalmol 2006;90:555-8.

16. Rizvi I, Celli JP, Evans CL, Abu-Yousif AO, Muzikansky A, Pogue BW, et al. Synergistic enhancement of carboplatin efficacy with photodynamic therapy in a three-dimensional model for micrometastatic ovarian cancer. Cancer Res 2010;70:9319-28.

17. Alander JT, Kaartinen I, Laakso A, Pätilä T, Spillmann T, Tuchin $\mathrm{W}$, et al. A review of indocyanine green fluorescent imaging in surgery. Int J Biomed Imaging 2012;2012:940585.

18. Achterberg FB, Deken MM, Meijer RPJ, Mieog JSD, Burggraaf J, van de Velde $\mathrm{CJH}$, et al. Clinical translation and implementation of optical imaging agents for precision image-guided cancer surgery. Eur J Nucl Med Mol Imaging 2021;48:332-9.

19. van Driel PB, van de Giessen M, Boonstra MC, Snoeks TJ, Keereweer S, Oliveira S, et al. Characterization and evaluation of the artemis camera for fluorescence-guided cancer surgery. Mol Imaging Biol 2015;17:413-23.

20. Gioux S, Choi HS, Frangioni JV. Image-guided surgery using invisible near-infrared light: fundamentals of clinical translation. Mol Imaging 2010;9:237-55.

21. Mishra A, Behera RK, Behera PK, Mishra BK, Behera GB. Cyanines during the 1990s: a review. Chem Rev 2000;100:19732012.

22. Jung W, Zhang J, Chung J, Wilder-Smith P, Brenner M, Nelson JS, et al. Advances in oral cancer detection using optical coherence tomography. IEEE J Sel Top Quantum Electron 2005;11:811-7.

23. Yashin KS, Kiseleva EB, Gubarkova EV, Moiseev AA, Kuznetsov SS, Shilyagin PA, et al. Cross-polarization optical coherence tomography for brain tumor imaging. Front Oncol 2019;9:201.
24. Hariri LP, Adams DC, Applegate MB, Miller AJ, Roop BW, Villiger $M$, et al. Distinguishing tumor from associated fibrosis to increase diagnostic biopsy yield with polarization-sensitive optical coherence tomography. Clin Cancer Res 2019;25:5242-9.

25. Hariri LP, Villiger M, Applegate MB, Mino-Kenudson M, Mark EJ, Bouma BE, et al. Seeing beyond the bronchoscope to increase the diagnostic yield of bronchoscopic biopsy. Am J Respir Crit Care Med 2013;187:125-9.

26. Chen Z, Zhao P, Luo Z, Zheng M, Tian H, Gong P, et al. Cancer cell membrane-biomimetic nanoparticles for homologoustargeting dual-modal imaging and photothermal therapy. ACS Nano 2016;10:10049-57.

27. Wang H, Li X, Tse BW, Yang H, Thorling CA, Liu Y, et al. Indocyanine green-incorporating nanoparticles for cancer theranostics. Theranostics 2018;8:1227-42.

28. Zheng M, Zhao P, Luo Z, Gong P, Zheng C, Zhang P, et al. Robust ICG theranostic nanoparticles for folate targeted cancer imaging and highly effective photothermal therapy. ACS Appl Mater Interfaces 2014;6:6709-16.

29. Mellor RH, Stanton AW, Azarbod P, Sherman MD, Levick JR, Mortimer PS. Enhanced cutaneous lymphatic network in the forearms of women with postmastectomy oedema. J Vasc Res 2000;37:501-12.

30. McLaughlin SA, Wright MJ, Morris KT, Giron GL, Sampson MR, Brockway JP, et al. Prevalence of lymphedema in women with breast cancer 5 years after sentinel lymph node biopsy or axillary dissection: objective measurements. J Clin Oncol 2008;26:5213-9.

31. Troyan SL, Kianzad V, Gibbs-Strauss SL, Gioux S, Matsui A, Oketokoun $\mathrm{R}$, et al. The FLARE intraoperative near-infrared fluorescence imaging system: a first-in-human clinical trial in breast cancer sentinel lymph node mapping. Ann Surg Oncol 2009; 16:2943-52.

How to cite this article: Shin YK, Eom JB. Optical imaging technology for real-time tumor monitoring. Med Lasers 2021;10:123-131. https://doi.org/10.25289/ML.2021.10.3.123 